\title{
USDA Animal Welfare Inspection Guide
}

\author{
B. Taylor Bennett, Andrew D. Cardon \& Matthew R. Bailey
}

On 2 December 2013, USDA Animal Care announced the release of the Animal Welfare Inspection Guide (AWIG), a reference document intended to assist APHIS inspectors and to ensure consistent inspections of facilities regulated under the Animal Welfare Act (AWA). This document is important to the biomedical research community, as it contains key information about the process inspectors should follow during inspections of regulated facilities. It also provides guidance regarding situations in which a citation should or should not be issued. A careful review of the AWIG can help research facilities evaluate their animal care and use programs to prevent citations for noncompliance.

On the same date the AWIG was announced, a second announcement was circulated in which the USDA informed the regulated community that the Agency is continuously trying to improve the way it enforces the AWA (http://content.govdelivery.com/accounts/USDAAPHIS/ bulletins/95b0d0). The announcement provided information on some changes that were being made with respect to the inspection process and the information that would be made available when inspection reports are posted online. With regards to documentation that will appear on the inspection reports, the Agency stated, "Inspection reports will now include the following information: 1) the citation for the most pertinent subsection of the regulations (as opposed to the full section); 2) a clear, detailed description of the noncompliant item; 3 ) an explanation of why the item is being cited as non-compliant and/or the impact the item is having on the animal(s); and 4) a deadline for when the non-compliant item needs to be corrected along with a general description of how the licensee/registrant can correct the item."

National Association for Biomedical Research, Washington, DC.
The AWIG contains 418 pages (its predecessor, the Consolidated Inspection Guide, contained 371 pages), nine chapters, four appendices and a glossary ${ }^{1}$. The overall style is very professional, and the document is easy to use in the downloadable PDF version. Particularly relevant aspects of the AWIG are discussed below.

Chapter 1-Introduction includes a disclaimer indicating that the AWIG is intended to be a reference document and that it does not supersede the AWA, AWA Regulations and Standards, the AC Policy Manual, the Required Inspection Procedures, standard procedures or the inspector's professional judgment.

Chapter 2-Required Inspection Procedures contains several sections of interest. One on Problems Addressed by the Facility Before Inspection indicates that a citation will not be written for a problem if all of the following statements are true: the problem was corrected in a timely manner; steps were taken to prevent the problem from recurring; there is no ongoing pattern of violations; and there were no serious animal welfare impacts associated with the problem. This section also includes a statement indicating that noncompliant items should be cited only in the most applicable section or subsection.

A section on Veterinary Care Direct Noncompliant Item (NCI) Identified requires inspectors to photograph each animal cited as part of a $\$ 2.33$ direct noncompliance.

A section on Inspection Photographs indicates that photographs must be taken to document Direct, Repeat and Critical NCIs (the term 'Critical' is not included in the glossary); Direct NCIs that have been corrected; any NCIs cited in a facility being investigated by Investigative and Enforcement Service (IES); NCIs likely to be appealed; and Vet Care NCIs involving animals when identified, reinspected or corrected. It also indicates that records documenting repeat, direct or transportation NCIs must be copied by some means. If they are copied by any means, the facility will be given $24-48$ hours to redact proprietary information from the records.

Chapter 3-General Inspection Procedures includes a section titled Type of Inspection; this information must be indicated on the inspection report. The term 'Routine Inspection' applies to a complaint, new or added site, partial or focused reinspection of Direct NCIs and search inspections. A section on the Correction Date contains a statement explaining that if an NCI is corrected during the inspection, the inspector may use his or her own discretion in deciding whether to cite it. If cited, the report will indicate that it was "corrected during the inspection."

An abbreviated section describing the Inspection Appeals Process instructs the inspector to provide a copy of the Appeals Process Fact Sheet during the inspection. It should be noted that when an appeal is successful, the amended report will have the original date of the inspection on the report itself, but the date the report was amended in the signature block.

Chapter 6-Veterinary Care contains a statement indicating that required veterinary records must be maintained for three years following the death or disposition of the animals and a list of required records to which this requirement applies, including acclimation statements for transportation, veterinary approved exemptions, health certificates for transport and outdoor housng approval.

Chapter 9-Animal Care Policies contains a relatively good Overview and Summary of Changes to the Policies. Appendix A contains various Forms and Worksheets, and Appendix B contains a table of sections with examples of Direct NCIs for each section included.

1. United States Department of Agriculture. Animal Welfare Inspection Guide (US Department of Agriculture, Riverdale, MD, 2013). 